\title{
Decomposition of 3D medical image based on Fast and Adaptive Bidimensional Empirical Mode Decomposition
}

\author{
OMAR AIT ZEMZAMI ${ }^{1}$, HAMID AKSASSE ${ }^{2}$, MOHAMMED OUANAN ${ }^{3}$, BRAHIM AKSASSE $^{4}$, \\ AZIZA BENKUIDER ${ }^{5}$
}

${ }^{1,2,3,4,5}$ Equipe ASIA, Computer Science Department, Moulay Ismaïl University, Faculty of Science and

Technology, P.O. Box 509, Boutalamine 52000 Errachidia, Morocco

E-mail: ${ }^{1}$ momarzemzami@yahoo.fr, ${ }^{2} h a k s a s s e @ y a h o o . f r,{ }^{3}$ ouanan_mohammed@yahoo.fr, 4baksasse@yahoo.com, ${ }^{5}$ abenkuider@hotmail.com

\begin{abstract}
Three-dimensional (3D) imaging and display have been subjects of much research due to their diverse benefits and applications. This paper presents a new approach for decomposing the three-dimensional medical images using Bidimensional Empirical Mode Decomposition (BEMD). The BEMD is an extension of the Empirical Mode Decomposition (EMD), which can decompose non-linear and non-stationary signals into basis functions called the Intrinsic Mode Functions (IMFs). IMFs are monocomponent functions that have well defined instantaneous frequencies. This decomposition, obtained by a process known as sifting process, allows extracting the structures at different scales and spatial frequencies with modulation in amplitudes and frequency. BEMD decomposes an image into bidimensional BIMFs. This paper suggests a simple, but effective, method for decomposing a three-dimensional medical image into basis function. This approach is neither parametric nor data driven, which means it does not depend on a priori basis set. Moreover, it preserves the totality of information in term of the quality of the reconstructed 3D image. The performance of this approach, using the BEMD, is approved with some medical images.
\end{abstract}

Keywords: Bidimensional Empirical Mode Decomposition (BEMD), Fast and Adaptive BEMD (FABEMD), Intrinsic Mode Function (IMF), 3D Reconstruction.

\section{INTRODUCTION}

The volume of medical images produced in the world is constantly increasing. Millions of threedimensional medical images (3D) are produced each year to diagnose or monitor a therapeutic effect. They provide data on the form and functioning of body organs. Unfortunately, these data are extremely difficult to exploit in a quantitative and objective manner [1].

The applications of three-dimensional reconstruction are numerous and varied: they extend from medicine [2], geography to the shipbuilding. In medicine, generally in the biomedical, reconstruction addresses the areas: anatomy [3, 4], electron microscopy and confocal [5], radiology [6], of surgery, cell biology, etc...

$3 \mathrm{D}$ reconstruction is particularly popular in microscopy. Indeed, the microscope lets to see very small structures but unfortunately provides only flat image. However, tissues, cells or subcellular structures have a three-dimensional architecture that the microscope cannot make it. The biologist is thus deprived of certain information. The scanning electron microscope overcomes this problem but it lacks flexibility and is limited in some cases (impossibility of seeing through the objects). In this case it is the $3 \mathrm{D}$ reconstruction takes over.

Three-dimensional reconstruction is also used in:

- Computer Aided Design: automotive, shipbuilding.

- Architecture and building technology: construction aid, study of the structure of materials (searching for cracks, grains, bubbles ...). 
O. A. Zemzami et al. / International Journal of Computer Networks and Communications Security, 1 (7), December 2013

- Geography: mapping (building of models from sections taken at different altitudes).

In medicine, three-dimensional reconstruction from serial sections is not a recent problem that dealt with new forms 3D acquisition modality. Some good manual 3D reconstruction techniques back to centuries before. They yield two different modes of reconstruction: graphic and rigid [7, 8]. Until now, several methods have been proposed to reconstruct a 3D model from structured data in 2D slices. The Marching Cubes algorithm proposed by W.E. Lorensen and H.E. Cline [9] and the algorithm based on the Delaunay triangulation proposed by J.D. Boissonnat and B. GEIGER [10] are references in this area.

Among the methods of image decomposition existing in the literature (for example wavelet [11]), Empirical Mode Decomposition (EMD) [12] is a flexible technique of signal decomposetion. EMD considers the signal to be processed on the scale of its local oscillations. The basic idea is to decompose a signal into a sum of components, called empirical mode or Intrinsic Mode Functions (IMFs). Each of IMF is a zero mean waveform, modulated in amplitude and frequency. It has a selfadaptive nature that enables it to identify changes in the signal, whether in terms of amplitude or frequency. Decomposition is not based on a predefined criterion, unlike the wavelet decomposition which requires a mother wavelet or kernel. Many researches proved that it is a very powerful tool for adaptive multi-scale analysis of non-stationary and non-linear signals, and took a place among the best known decomposition filters. The EMD is successfully applied in many fields ranging from pure scientific context to engineering $[13,14,15,16,17,18,19$, $20,21]$.

This decomposition technique has also been extended to new ones that analyze bidimensional images. They are known by Bidimensional EMD (BEMD), image EMD (IEMD), 2D EMD etc [22, 23]. Interpolation is an important issue both in the field of image signal for the estimation and the extraction of the IMFs from extrema. Different procedures can be used to calculate average envelope [12]. In FABEMD (Fast and Adaptive BEMD), order statistics filters are employed to estimate the envelope surfaces from the data instead of surface interpolation.

In this paper, we propose an extension of BEMD for three-dimensional medical images based on 3D reconstruction. The 3D decomposition schemes process the data as multi 2D slices with BEMD like technique. The main conceptual innovations are the introduction of Intrinsic Mode Functions based on global properties of the $3 \mathrm{D}$ medical image which makes the image sequence into a set of modes.

\section{BIDIMENSIONAL EMPIRICAL MODE DECOMPOSITION}

EMD is an adaptive decomposition of signals [24], introduced by Huang [12] for one-dimensional data and then extended to Bidimensional signals.

The novel BEMD approach is a highly adaptive decomposition [25]. It is based on the characterization of the image with this decomposition in Intrinsic Mode Function (IMF) where the image can be decomposed into a redundant set of composite images called IMFs and a residue. An IMF is characterized by two specific properties [26]:

- The number of zero crossing and the number of extrema points is equal or differs only by one.

- It has a zero local mean.

The algorithm is described as follows:

Given an image $I$

1. Fixed. $\varepsilon, i=1, r_{i-1}=I$ (residue)

2. Extraction of the $i^{\text {th }} \mathrm{IMF}$ :

a. Initialization: $h_{i, j-1}=r_{i-1}, j=1$

b. Extract local minima and maxima of $h_{i, j-1}$

c. Interpolate the local extrema to construct the upper and the lower envelope respectively, $\mathrm{U}_{i, j-1}$ and $\mathrm{L}_{i, j-1}$

d. Calculate the average of the two envelopes:

$$
m_{i, j-1}=\frac{U_{i, j-1}+L_{i, j-1}}{2}
$$

e. Update:

$$
h_{i, j}=h_{i, j-1}-m_{i, j-1}, j=j+1
$$

f. Calculate the stopping criterion :( Standard Deviation)

$S D=\sum_{x=1}^{M} \sum_{y=1}^{N} \frac{\left|h_{i, j}(x, y)-h_{i, j-1}(x, y)\right|^{2}}{\left|h_{i, j-1}(x, y)\right|^{2}}$ 
O. A. Zemzami et al. / International Journal of Computer Networks and Communications Security, 1 (7), December 2013
g. Decision: Repeat steps (b) to (f) until $S D(j) \leq \varepsilon$, and then put $I M F_{i}=h_{i, j}\left(i^{\text {eme }} I M F\right)$

3. Update residual:

$$
r_{i}=r_{i-1}-I M F_{i}
$$

4. Repeat steps 2 with $i=i+1$ until the number of extreme in $r_{i}$ is less than 2 .

\subsection{Image extrema extraction}

The first step of the sifting process in the BEMD is to locate the local extrema points (maxima and minima) of the image intensity. The simple way is to use the pixel neighbouring information in the image; such that a point is maxima (respectively minima) if its value is strictly higher (respectively strictly lower) than all its 8connected closer neighbours.

\subsection{Surface interpolation}

The interpolation is considered as a delicate stage in the 2D-sifting process it has a remarkable influence on the results of the decomposition. Several techniques have been proposed using, for instance, radial basis functions such as thin-plate splines [26, 27, 22, 28], smoothing spline technique [29]. BEMD, however, requires very high computational cost [22].

\subsection{Stopping criterion for the sifting process}

The sifting process in the BEMD decomposition consist on decomposing an input signal into set of functions defined by the signal itself, these functions are called BIMFs. A BIMF is characterized by some specific properties.

Each BIMF is expected to have the following properties:

- The number of zero crossings and the number of extrema points is equal or differs by only one.

- The envelopes defined by the local maxima and minima, respectively, are locally symmetric around the envelope mean.

The sifting process stops when the resulting image satisfied the characteristics of a BIMF as it is described above. In other words, it stops when the envelope mean signal is close enough to zero. After a BIMF is found, we define the residue as the result of subtracting this BIMF from the input image and then we iterate on the residue. The BEMD is completed when the residue, ideally, does not contain any extrema points. This is if we suppose that the sifting process will converge, things that never have been rigorously demonstrated. Then, we should to determine a criterion to stop sifting process; this can be accomplished by limiting the size of the SD which is computed from the two consecutive sifting results. In practice, we have used SD between 0.02 and 0.3 and this stop criterion gives satisfying results.

\subsection{Stopping criterion for the decomposition}

Generally, the decomposition stops when the number of extrema is less than 2. This means that there are no more oscillations to extract. In some cases we can have a high number of IMFs without meeting this condition, so we should set a maximal number of IMFs to extract. We can stop the decomposition depending on the need; for example, in image denoising we will need only the first IMF.

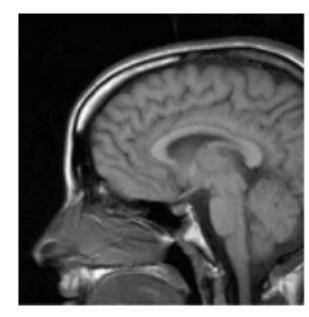

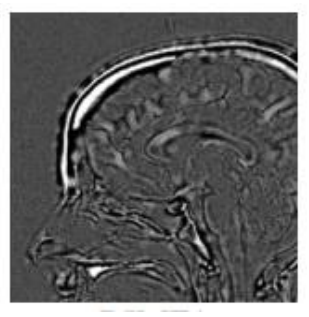

BIMF1

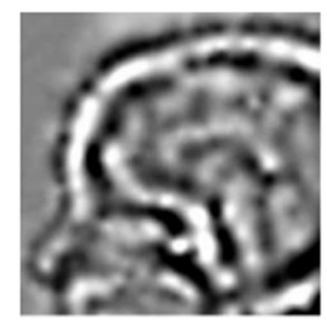

BIMF3

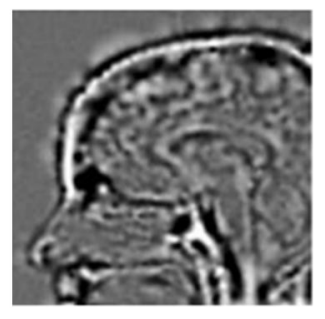

$B I M F 2$

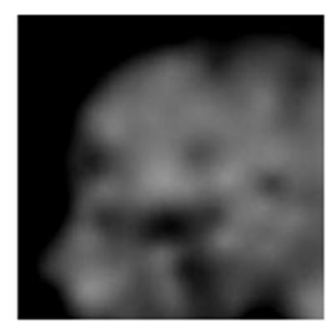

Residue
Fig. 1. Decomposition of a medical image using BEMD 
3 FAST AND ADAPTIVE BEMD (FABEMD)

The so called FABEMD, proposed by sharif. $M$ et al [30], is a new version of the bidimensional empirical mode decomposition. It is simple, effective, fast and adaptive.

FABEMD decomposition (Fast and Adaptive BEMD) has the same steps as BEMD except that the building envelope average is different from that of the BEMD.

\section{Algorithm}

FABEMD differs from the original algorithm BEMD, mainly in the evaluation process of the upper and lower envelopes and in limiting the number of iterations for each BIMF. This change makes the BEMD faster. This is due to the fact that the interpolation step is replaced by spatial processing followed by a smoothing operation. The treatment uses two operators:

1) a filter Max to estimate the envelope Max

2) a filter Min to estimate the envelope Min

The size of the filter is determined from the extrema matrix Max and Min.

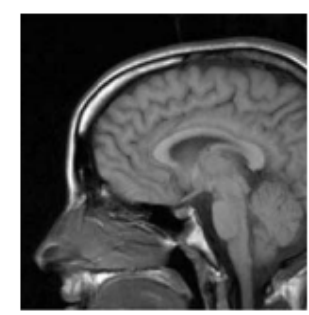

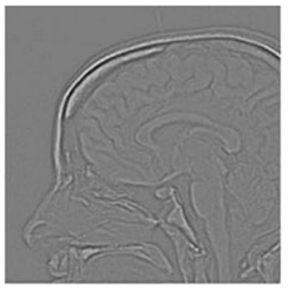

BIMF1

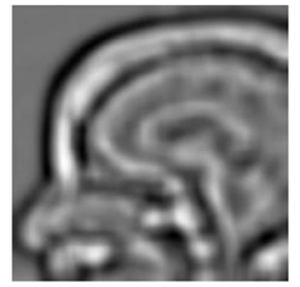

BIMF3

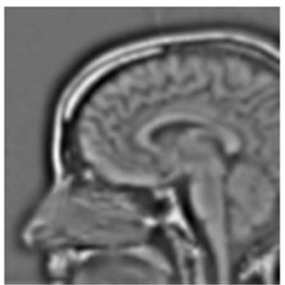

BIMF2

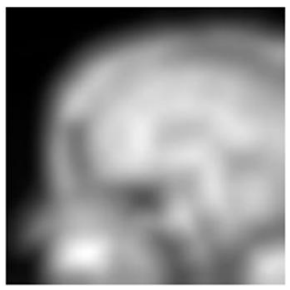

Residue
Fig. 2. Decomposition of a medical image using FABEMD
The BIMFs obtained by FABEMD are easier to interpret. On the other hand, contours at different scales are visible on BIMFs obtained by FABEMD. In [30], a study comparing BEMD and FABEMD was presented in detail. It showed that the FABEMD provides a more accurate evaluation of the BIMFs that the BEMD.

\section{THREE-DIMENSIONAL RECONSTRUCTION FROM SERIAL SECTIONS}

Some technologies are only able to obtain that the surface of the object to be reconstructed as the stereo vision method and the method of the characteristic points. So if you want to explore more than the surface of the object, it is necessary to make sections in the object to be reconstructed. This is the method that is commonly used in medicine and biology. The reconstruction methods depend on the process for obtaining the slices.

The slices can be real or virtual:

- The real slices are obtained by cutting the object physically; this is the case in cytology where cells are sectioned by ultramicrotomy.

- The virtual slices are obtained by using physical properties, acoustic or magnetic of various materials (tissue, bone). This is the case with the Computed tomography (CT), ultrasound, MAGNETIC RESONANCE IMAGING, electron and confocal microscopy.

If the slices are parallel, we say that they are serial sections. And if they are radial slices, we say that they are concentric sections. The real sections are those representing the intersection of the cutting plane and the object. The sections can be projections; in this case it is an accumulation of data taken on lines perpendicular to the section plane (measurement of the intensity resulting from crossing a tissue by an X-ray, for example). It was those kinds of sections we found in Nuclear Magnetic Resonance, Radiography and Computed tomography. The reconstruction used in this case is described by R.Gordon and G.T.Herman [31].

In the case of the surface reconstruction, the focus is only on the surface, and in case of the volume reconstruction, it is the object as a whole is interesting. In some cases only one visualization is used; contrariwise, in other cases an analysis and description of the object are needed to well understand the three-dimensional structure of the object. The type of representation depends on the 
use of the $3 \mathrm{D}$ object. If the user wants a simple visualization, the surface is sufficient; this is what usually occurs in CAD applications. If the object must constitute a data of a description process, modeling or analysis of 3D objects, it will need whole object. In the general case, the aim is much more than the description of three-dimensional structures encountered as the visual rendering.

Cross sections are increasingly used in many fields to represent 3D objects particularly in medical imaging. But, 3D sampled images do not reflect the real three-dimensional aspect of the object; they simply give a limited and partial view.

The system we propose consists mainly of six steps: acquisition, preprocessing, registration, segmentation, model generation and finally visualization. Second and third stages is somehow preparatory steps for the overall reconstruction process. That is why we have grouped them together to a single step.

\subsection{Acquisition step}

The information captured by the physical device (a CT scan, MRI or US) is a measure of some physical properties of the body. The result of this step is a sequence of 2D images, usually grayscale, forming the cuts.

\subsection{Preprocessing and registration step}

On acquisition, external conditions can intervene to affect the quality of the acquired image. The lighting conditions, the level of parasitic signals, nature of the atmosphere (dust, humidity ...), the sampling resolution of the scanner, camera calibration ... All these phenomena add other information called noise. Then, as first phase, we should remove the noise without affecting the useful information. After that, we proceed to improve the quality of the image to facilitate the work of later stages. For this, many studies in the literature offer an extensive range of preprocessing techniques.

In the second phase, the registration of sections takes place. Its role is to realign the cuts in order to get a shape similar to the original subject. In fact, this is necessary where the conditions for obtaining the sequence differ from one section to another, allowing some sections to be rotated or to submit on any deformation. In the case where the sequence consists of sections MRI, CT or US they are aligned, and therefore this step is omitted.

\subsection{Segmentation Step}

Segmentation is a process to decompose the images sequence into a set of homogeneous regions. Its role in the reconstruction process is to locate the volume of interest (VOI) within the set of sections. Otherwise, the set of pixels representing the same tissue type in a section will be grouped to form a single region, which offers major interest to the practitioner.

A second task assigned to this step is the identification or labeling of the various segmented VOI. Indeed, the segmented regions belonging to the same type of tissue should then be classified according to their anatomical natures. For example, isolating the left ventricle of on the right, or the veins of different arteries. The result is a sequence of binary images where the binary value of each pixel indicates whether it belongs to the VOI. The binary nature of the images greatly reduces the amount of data to be processed.

Another task assigned to this step is to extract, from the volume of interest obtained, all the contours that delimit it, along the sequence of binary images. The final result of this step is a sequence of closed contours plans.

\subsection{Modeling Step}

This step is the core of the reconstruction process since it builds three-dimensional information. The goal of reconstruction is to provide a geometric description of the digitized object, based mainly on the calculation of a model by volume or by surface which will be an approximation of the set of data. This operation is decomposed into two steps [32]:

- Global connection

- Local connection

The final result of this step is a $3 \mathrm{D}$ model, consisting of a mesh, approximating the initial object.

\subsection{Visualization step}

This is the final step. Visualization is the projection process the $3 \mathrm{D}$ model obtained on a $2 \mathrm{D}$ plane consisting of the screen for example. This projection should provide the maximum possible information contained in the model, and should also enable an exploration or navigation of the information density presented by this model. In addition, it should allow for intuitive and flexible interaction for user-machine. 
O. A. Zemzami et al. / International Journal of Computer Networks and Communications Security, 1 (7), December 2013

In this work, we focused on the third step (segmentation) and the fourth stage (the generation of the 3D model).
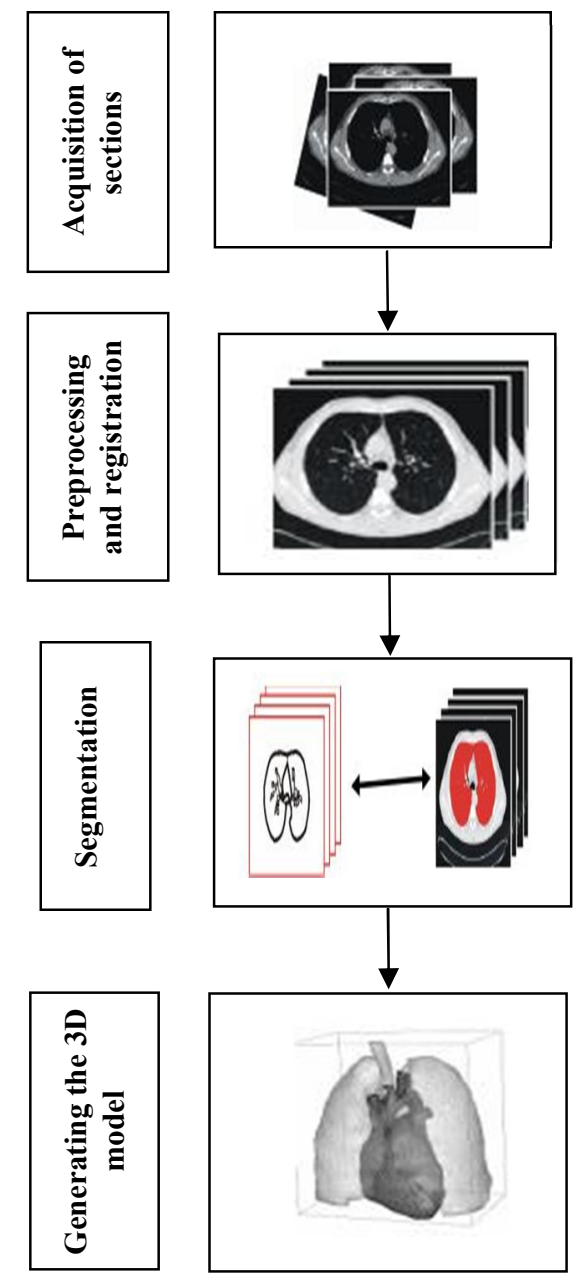

Fig. 3. Principle of the proposed three-dimensional reconstruction system

\section{BEMD APPROACH FOR MEDICAL IMAGES}

Our approach is based on the application of the decomposition FABEMD for each 2D image in a series of sections. We obtain a set of IMFs and a residue for each image. With the $3 \mathrm{D}$ reconstruction of each series of IMFs, we obtain the result image (3D IMF).
The process decomposition approach with BEMD is illustrated in figure 4.

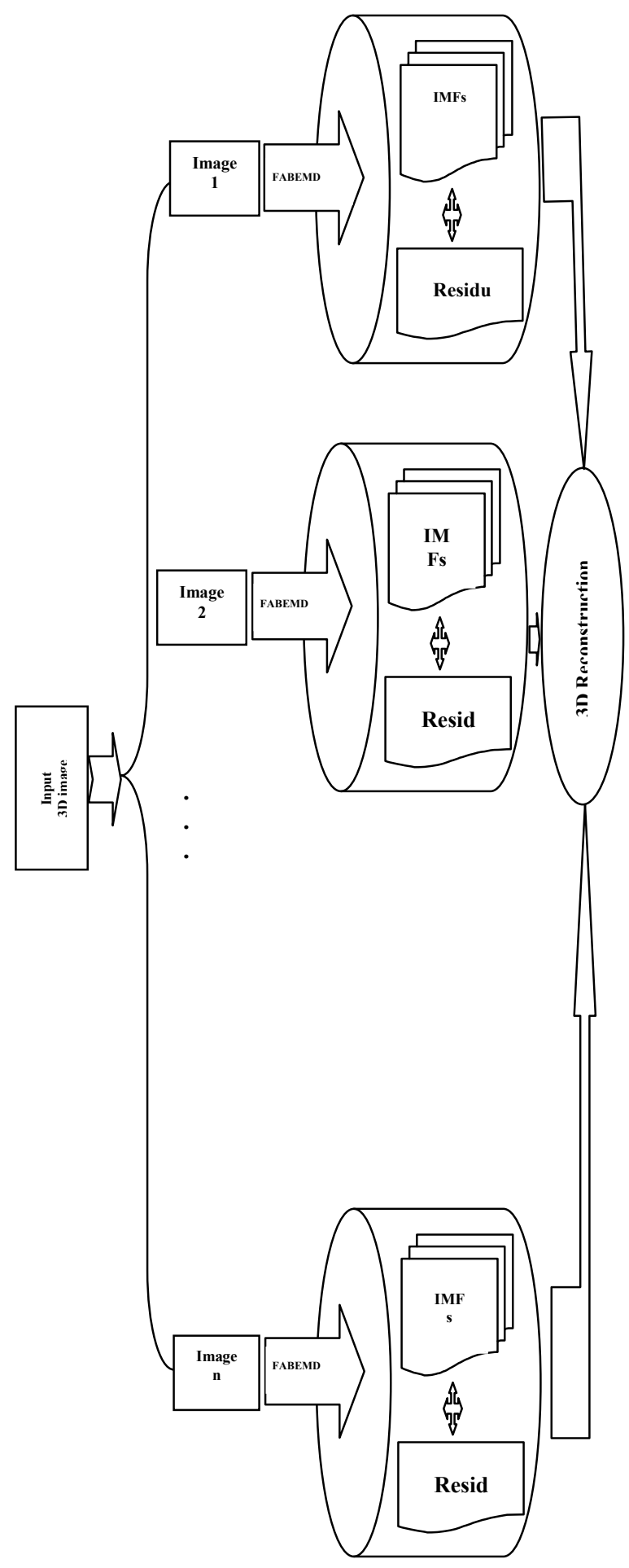

Fig. 4. The BEMD approach for 3D Medical Image 
O. A. Zemzami et al. / International Journal of Computer Networks and Communications Security, 1 (7), December 2013

In this section we use a series of 55 CT image (Fig. 5) of a pelvis (Fig. 6).

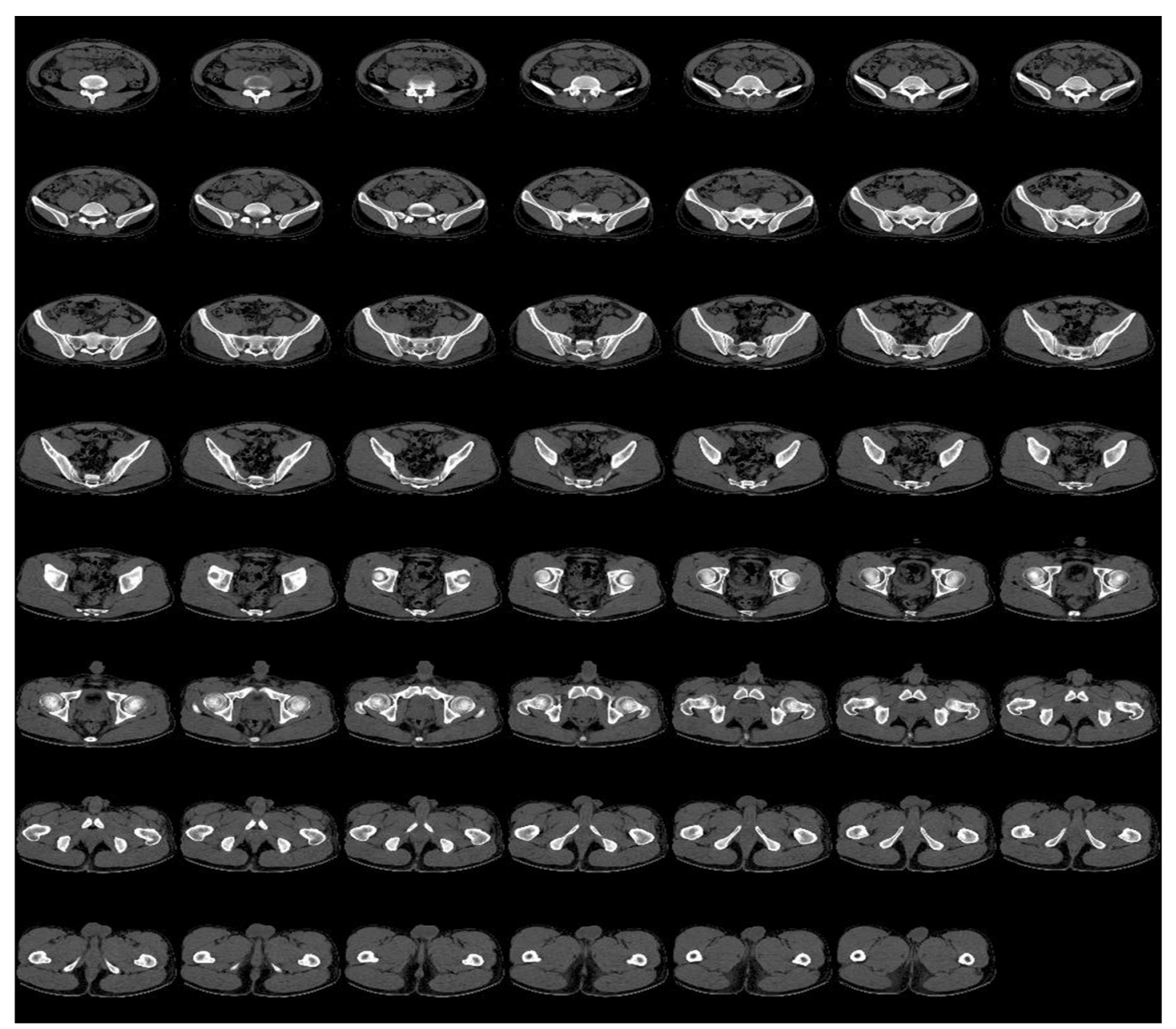

Fig. 5. Series of 55 CT image ordered from left to right and from top to bottom

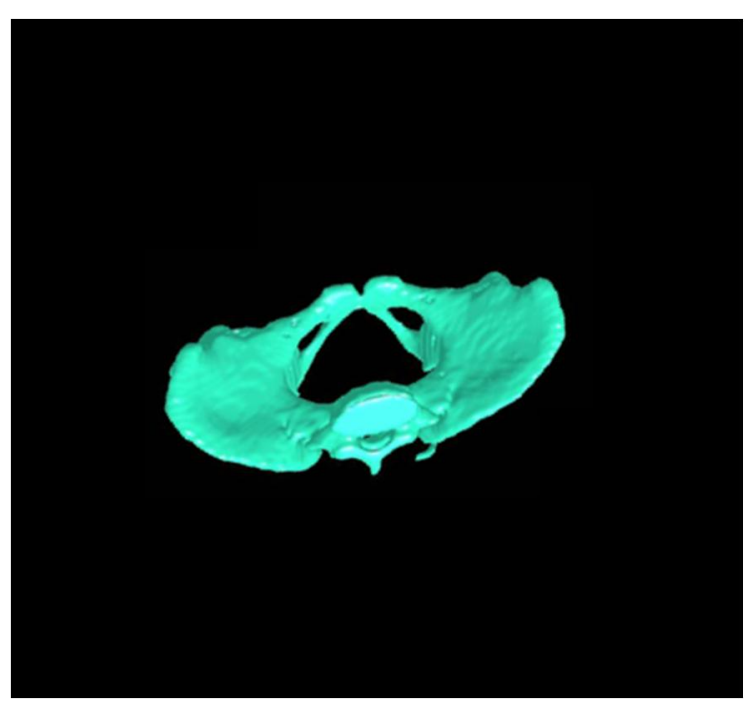

Fig. 6. 3D image of the pelvis 
O. A. Zemzami et al. / International Journal of Computer Networks and Communications Security, 1 (7), December 2013

Each image of the previous series is subject to the decomposition FABEMD (Fig. 7).

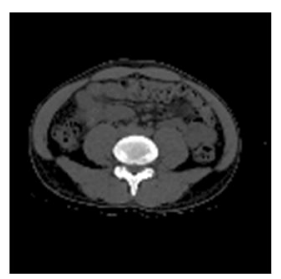

Image1

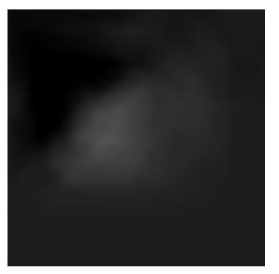

IMF5

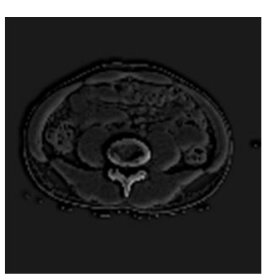

$I M F 1$

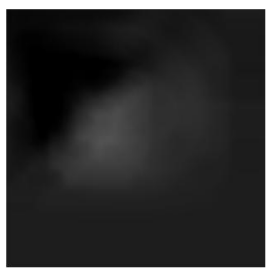

IMF6

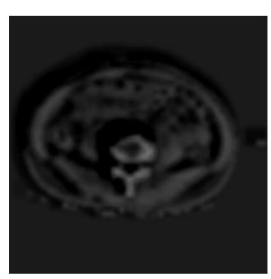

$I M F 2$

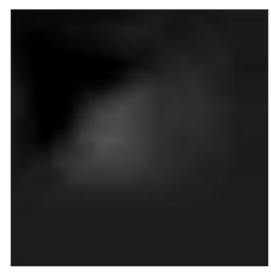

IMF7

$\bullet$

$\bullet$

$\bullet$

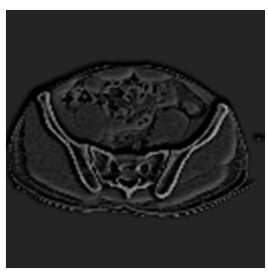

$I M F 1$

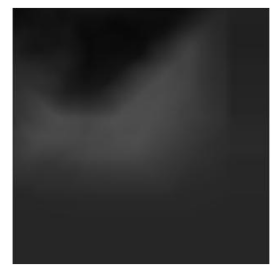

IMF6

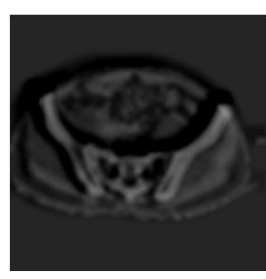

$I M F 2$

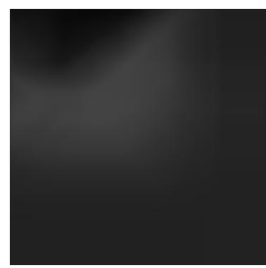

IMF7

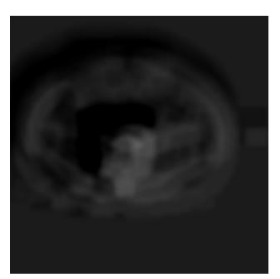

$I M F 3$

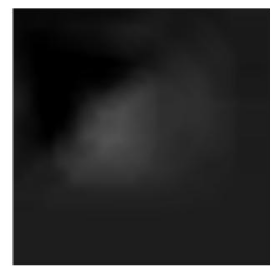

$I M F 8$

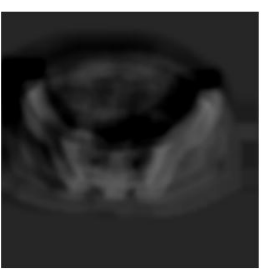

$I M F 3$

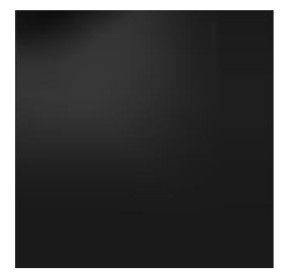

IMF8

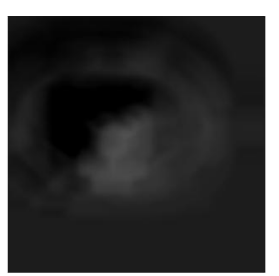

IMF4

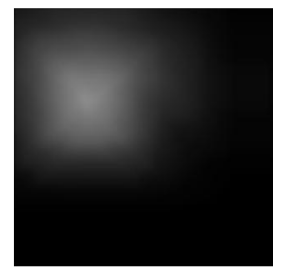

Residue

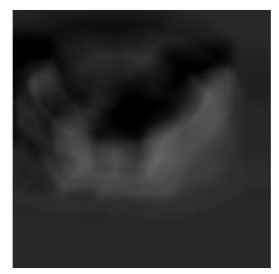

IMF4

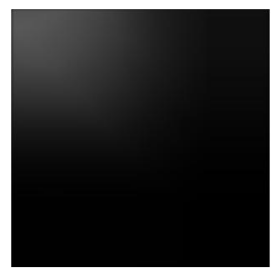

Residue 
O. A. Zemzami et al. / International Journal of Computer Networks and Communications Security, 1 (7), December 2013

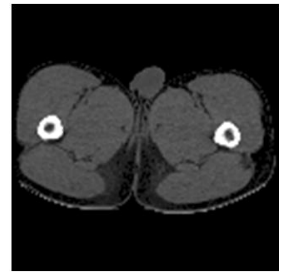

Image 55

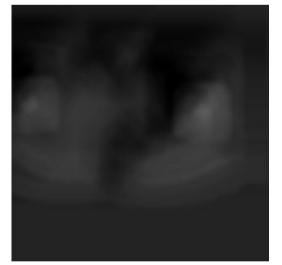

IMF5

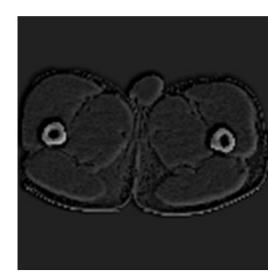

$I M F 1$

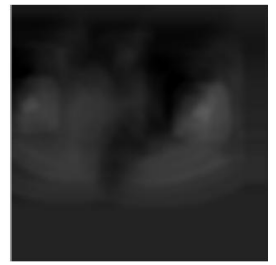

IMF6

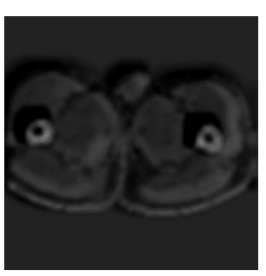

$I M F 2$

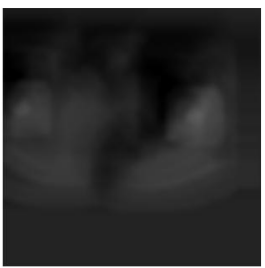

$I M F 7$

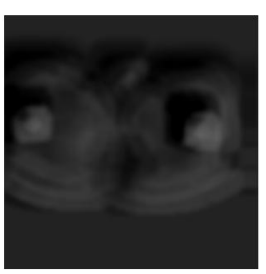

$I M F 3$

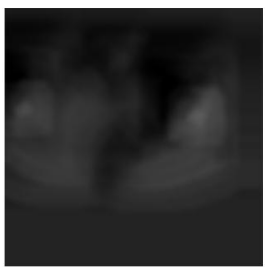

$I M F 8$

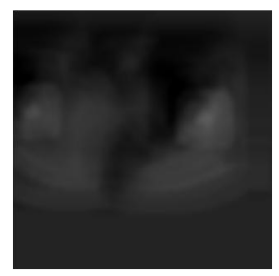

IMF4

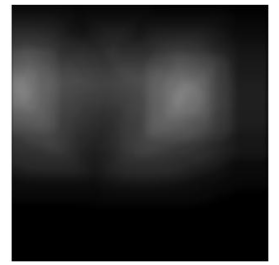

Residue

Fig. 7. The result of the decomposition with BEMD for each image in the series of sections

In general, two images do not have the same number of IMFs. If it is the case and in order to construct the 3D IMFs, we duplicate the final IMF of images that have a depth of decomposition inferior than others. For example, in figure 7, image 1 has in fact four IMFs, whereas the image 17 has 8
IMFs which correspond to the maximum depth between the ones of all images of the series. Therefore, to ensure the 3D reconstruction of IMFs we place IMF8 = IMF7 = IMF6 IMF5 $=$ IMF4 and so for all the images that have a depth inferior to 8 .

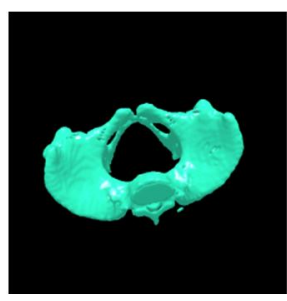

3D image

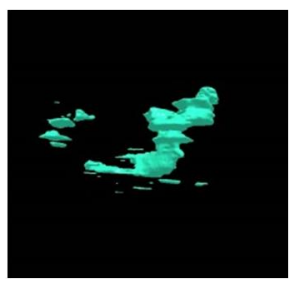

3D IMF5

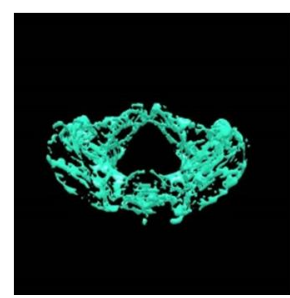

3D IMF1

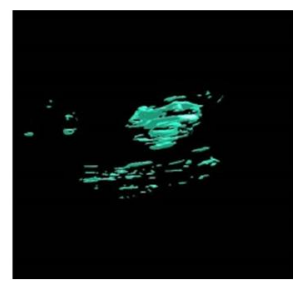

3D IMF6

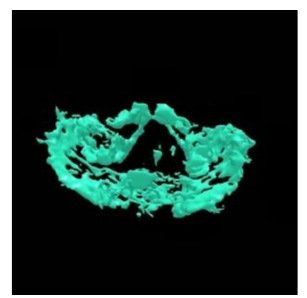

3D IMF2

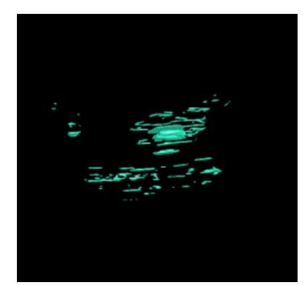

3D IMF7

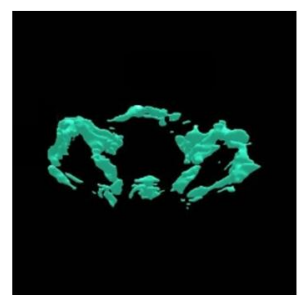

3D IMF3

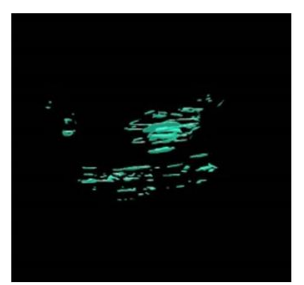

3D IMF8

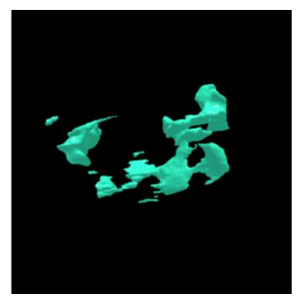

3D IMF4

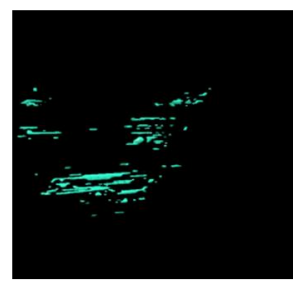

Residue
Images in the figure 8 shows the results obtained after the 3D reconstruction of each series of IMFs and the series of the residues.

\section{CONCLUSION}

In this work, we have presented a technique for implementing the BEMD for medical images decomposition. BEMD decomposition which has been proven in the case of the 1D signal analysis and has proven a powerful tool for analyzing and interpreting images, its application extend from watermarking, indexing to the image compressing.

The aim of the work was to decompose 3D medical images into 3D IMFs. The medical image can be characterized by global information extracted from the 3D IMFs. We focused on the 
problem of 3D reconstruction of 2D images. Threedimensional reconstruction of objects from serial sections allows for a shift from model previously scanned to a 3D mathematical model reflecting, at the maximum possible, the main properties of the object. This was primarily motivated by the incapacity of $2 \mathrm{D}$ imaging to provide a sufficient understanding of both the complexity of the studied objects and the necessity of having a 3D computer representation of many real objects. Problem, widely reported in military applications, industrial, scientific and especially medical, known as 3D reconstruction of serial sections.

As a perspective, we will deal with this problem in its $3 \mathrm{D}$ form.

\section{REFERENCES}

[1] N. Ayache, "Automatic analysis of medical images: state of the art and perspectives (L'analyse automatique des images médicales : état de l'art et perspectives )“", Research Report No. 3364 - EPIDAURE project INRIA - February 1998.

[2] P. Cook, "Three-dimensional reconstruction from serial sections for medical application", Ph. D. University of Missouri-Columbia. 1980.

[3] C. Barrillot, "Methods of three-dimensional reconstruction and computer graphics applied to the representation of morphological structures in medicine (Méthodes de reconstruction tri-dimensionnelle et de synthèse d'images appliquées à la représentation des structures morphologiques en médecine)“, Thesis, University of Rennes I. December 1984.

[4] J. Sequeira, "Interactive modeling of objects with complex shapes from heterogeneous data (Modélisation interactive d'objets de forme complexe à partir de données hétérogènes)“, Thesis, University of Franche-Comté, Besançon. November 1987.

[5] F. Macias-Garza, K.R.Diller, A.C.Bovik, S.J.Aggarwal, J.K. Aggarwal, "Obtaining a solid model from optical serial sections", Pattern Recognition, vol 22, No 5.1989, pp 577-586.

[6] G.G. Pieroni, Y.P. Wing, "Reconstructing the activity surface for cardiac PET imagery", 9th International Conference on Pattern Recongnition, Rome. Novembre 1988, pp 125133.

[7] G.Chinga, "Three-dimensional reconstruction of plant cells exposed to different gravity conditions", Master of Science thesis,
Norwegian University of Science and Technology, 1997.

[8] S. Lozanoff and D. Moody, "A brief review of computerized 3D reconstruction of anatomical structures", Internal report, University of Alberta - 1998 .

[9] W.E. Lorensen and H.E. Cline, "Marching cubes: a high resolution $3 \mathrm{~d}$ surface reconstruction algorithm", Computer Graphics, 21(4), 163-167; July 1987.

[10]J.D. Boissonnat and B. GEIGER, "Three dimensional reconstruction of complex shapes sased on the Delaunay triangulation", Research report $\mathrm{N}^{\circ}$ 1697, INRIA- Sophia Antipolis 1992.

[11]S.G. Mallat, "A theory for multiresolution signal decomposition: the wavelet representation", IEEE Trans. Pattern Analysis and Machine Intelligence, Vol 11, No7. 1989, pp. 674-693.

[12] N. Huang, Z. Shen, SR. Long, MC. Wu, HH. Shih, Q. Zheng, N-C. Yen, CC. Tung and HH. Liu, "The empirical mode decomposition and the Hilbert spectrum for non-linear and nonstationary time series analysis", Proc R Soc Land Ser A 454 1998,pp. 903-995.

[13]R. Kazys, D. Pagodinas, and O. Tumsys, "Application of the hilbert-huang signal processing to ultrasonic nondestructive testing of composite materials," Ultragarsas, vol. 50, no. 1, pp. 17-22, 2004.

[14]P. J. Oonincx and J. P. Hermand, "Empirical mode decomposition of ocean acoustic data with constraint on the frequency range", in Proceedings of seventh European Conference on Underwater Acoustics, Delft, The Netherlands, 5-8 July 2004.

[15]Z. Wu and N. E. Huang, "A study of the characteristics of white noise using the empirical mode decomposition method," The Royal Society, vol. 460, pp. 2597-1611,2004.

[16]H. Liang, S. L. Bressler, E. A. Buffalo, R. Desimone, and P. Fries, "Empirical mode decomposition of field potentials from macaque v4 in visual spatial attention," Biological Cybernetics, vol. 92, pp. 380-392, 2005.

[17] Y.-M. Mao and P.-W. Que, "Application of hilbert-huang signal processing to ultrasonic non-destructive testing of oil pipelines," Journal of Zhejiang University Science A, vol. 7, no. 2, pp. 130-134, 2006.

[18] M. K. I. Molla, A. Sumi, and M. S. Rahman, "Analysis of temperature change under global warming impact using empirical mode decomposition," International Journal of 
O. A. Zemzami et al. / International Journal of Computer Networks and Communications Security, 1 (7), December 2013

Information Technology, vol. 3, no. 2, pp. $131-139,2006$.

[19]C. Cai, W. Liu, J. S. Fu, and Y. Lu, "A new approach for ground moving target indication in foliage environments," Elsevier Signal Processing, vol. 86, pp. 84-97, 2006.

[20]J. Cexus and A. Boudraa, "Nonstationary signals analysis by teager- huang transform(tht)," in EUSIPCO, Florence, Italy, 2006.

[21] S. Benramdane, J. C. Cexus, A. O. Boudraa, and J. A. Astolfi, "Time- frequency analysis of pressure fluctuations ona hydrofoil undergoing a transient pitching motion using hilbert-huang and teager-huang transforms", in ASME PVP 2007/CREEP 8, San Antonio, Texas, USA, July 22-26 2007.

[22] J.C. Nunes, Y. Bouaoune, E. Deléchelle, O. Niang, and $\mathrm{Ph}$. Bunel, "Image analysis by bidimensional empirical mode decomposition" Image and Vision Computing, vol. 21, no. 12, pp. 1019-1026, 2003.

[23]Z. Liu, H. Wang, and S. Peng, "Texture classification through directional empirical mode decomposition", Proceeding of th 17 th IEEE International Conference on Pattern Recognition (ICPR'04), vol. 4, pp. 803-806, Cambridge, UK, August 2004.

[24]A. Linderhed, " Image empirical mode decomposition: a new tool for image processing" Advances in Adaptive Data Analysis 1(2) 2009, pp. 265-294.

[25]F. Ben Arfia, M. Ben Messaoud and M. Abid, "A new image denoising technique combining the empirical mode decomposition with a wavelet transform technique", 17th International Conference on Systems, Signals and Image Processing 2010.

[26]A. Linderhed, "2D empirical mode decompositions in the spirit of image compression", Proc. of SPIE, Vol. 4738 2002, pp.1-8.

[27] A. linderhed, “Adaptive Image Compression with Wavelet Packets and Empirical Mode Decomposition", Phd thesis, Link"oping Studies in Science and Technology, 2004.

[28]J.C Nunes, “Analyse Multiéchelle d'image. Application à l'Angiographie Rétinienne et à la DMLA", PhD Thesis, Université. Paris 12, décembre 2003.

[29] Donghoh, K., and Hee-Seok, O., "EMD: A Package for Empirical Mode Decomposition and Hilbert Spectrum", The R Journal, Vol-1/1, May 2009.

[30] Sharif M. A. Bhuiyan, Reza R. Adhami, Jesmin F. Khan, "Fast and adaptive bidimensional empirical mode decomposition using order-statistics filter based envelope estimation," EURASIP Journal on Advances in Signal Processing, Special Issue on the Empirical Mode Decomposition and the Hilbert-Huang Transform, Article ID 728356, pp. 1-18, 2008.

[31]R. Gordon, G.T. Herman, "Three-Dimensional reconstruction from projections: a review of algorithms", Intern.Rev.of Cytology, 38.1974, pp 111-151.

[32]B. Geiger, "Three dimensional modelling of human organs and its application to diagnosis and surgical planning", INRIA Rapports de Recherche, No. 2105, 1993. 